\title{
Sectional Parametric Design and Aerodynamic Performance Estimation In Small Aircraft
}

\author{
Yesuenyeagbe A.K. Fiagbe ${ }^{1}$, Fredrick Ferguson ${ }^{2}$, and Lawrence E. Ansong ${ }^{1}$ \\ ${ }^{I}$ Department of Mechanical Engineering, College of Engineering, KNUST, Ghana, \\ Email:yakfiagbe@yahoo.com,yakfiagbe.soe@ knust.edu.gh \\ ${ }^{2}$ Department of Mechanical Engineering, College of Engineering, NCA\&TSU, USA
}

\begin{abstract}
Small aircraft in the form of personal or remote controlled aircraft design could be approached in such a way as to derive optimum performance for any configuration that may be developed for given mission. In practice, the aerodynamic performance is estimated from the coefficients obtained due to the chosen wing profile. However, it can be envisioned that every section of the configuration could contribute to the aerodynamic forces. In this paper a technique or method that takes into account the contribution of all sections of the configuration with the aim of improving the accuracy of the evaluation of the aircraft performance is developed. The concept of the method is built on object oriented technique. Input parameters comprise of two kinds, namely, fixed and design parameters. The output gives the geometry and performance characteristics of the aircraft. The performance is modelled as integral function over the surface and results in Lift and Drag forces. The lift performance of models with same wing profile indicated that fuselage components also contributed, about $10 \%$ at $2^{\circ}$ angle of attack, toward the overall lift force. The total lift and drag estimates of an aircraft using the developed system is more accurate than using estimates from the wing alone.
\end{abstract}

Key Words: - Parametric design, Performance, small Aircraft, Lift, Drag

\section{INTRODUCTION}

Since the successful flight of aircraft by the Wright brothers, air transportation has seen tremendous revolution and has become very important means of transportation. Small aircrafts may be in the form of personal, remote or radio controlled aircrafts likewise has also become essential. These usually operate at low speed and are used for different kinds of activities.

Air vehicle design requires a multidisciplinary perspective and involves knowledge in a wide range of subjects such as aerodynamics, engineering structures, propulsion, control systems, and materials. According to Stinton, (1998), the configuration or shape is influenced by the aerodynamics, propulsion and structural design. As such any aircraft configuration must be aerodynamic, structurally sound and provide for propulsion system for given mission.

The lift, aerodynamic force normal to the direction of free-stream flow, is typically estimated from parameters associated with pre-determined wing geometry. However, it can be envisioned that every section of the configuration could contribute to the aerodynamic forces. This research developed a technique or method that takes into account the contribution of all sections of the configuration with the aim of improving the accuracy of the evaluation of the aircraft performance. Labrujere and Slooff (1993) used the inverse design approach for the aerodynamic design of the aircraft. The approach was mainly for the wing design and employed computational methods. Turevskiy et al, (1999) used a combination of free and commercial off-theshelf (COTS) modelling and simulation software to simplify and improve the flight vehicle design process. Raymer (2002), in his doctoral thesis, used Multidisciplinary Optimization (MDO) technique to enhance the conceptual design process of aircraft design. He employed various techniques such as orthogonal steepest descent (full factorial stepping search), Monte Carlo and mutation-based evolutionary option to develop a design code. Neufeld et al (2007) developed Multi-Objective Genetic Algorithm (MOGA) optimizer to assist in the design process for very light jet (VLJ) and an Unmanned Aerial Vehicles (UAVs). In all these cases, the aerodynamic performance estimate was based on the wing geometry and coefficients to determine the lift and drag.

One of the initial significant processes in aircraft design is the development or generation of the configuration. Presently, geometry generation methods usually involve the use of computer-aided design (CAD) software, although there are some examples of parametric geometry tools such as Boeing's proprietary tool, GGG1,2 (General Geometry Generator), NASA's RAM3,4 (Rapid Aircraft Modeler) tool (Gloudemans at el, 1996), and Avid's PAGE5 (Parametric Aircraft Geometry Engine) application. Unfortunately, CAD-based modeling can be detrimental to efficiency as geometry must usually be generated manually and then converted to something usable by an analysis method. Generating the initial geometry model in CAD can be tedious and 
time-consuming. Rodriguez and Sturdza (2006) developed the Rapid Geometric Engine (RAGE) using some defined parameter. RAGE is good for shapes operating at Mach numbers above 0.5.

\section{METHODOLOGY}

Object-oriented technique is used in the development of aircraft configuration and determining performance of each object or section of configuration, leading to the overall aerodynamic performance. The aircraft configuration was decomposed into components referred to as objects (figure 1) and mapped into objectoriented environment. Development of the design parameters was carried out to completely describe the aircraft geometry. These parameters independently describe various objects. For the purpose of aerodynamic analysis, numerical wind tunnel was developed. Aerodynamic analysis was conducted in the numerical wind tunnel. The result of the numerical wind tunnel tests was used in estimating the aerodynamic performance of the aircraft. Finally, parametric studies were conducted on the defined objects using the design parameters.

The system is developed using FORTRAN 95 programming language. The results of objects created are visualized using TECPLOT which is post data processing software.

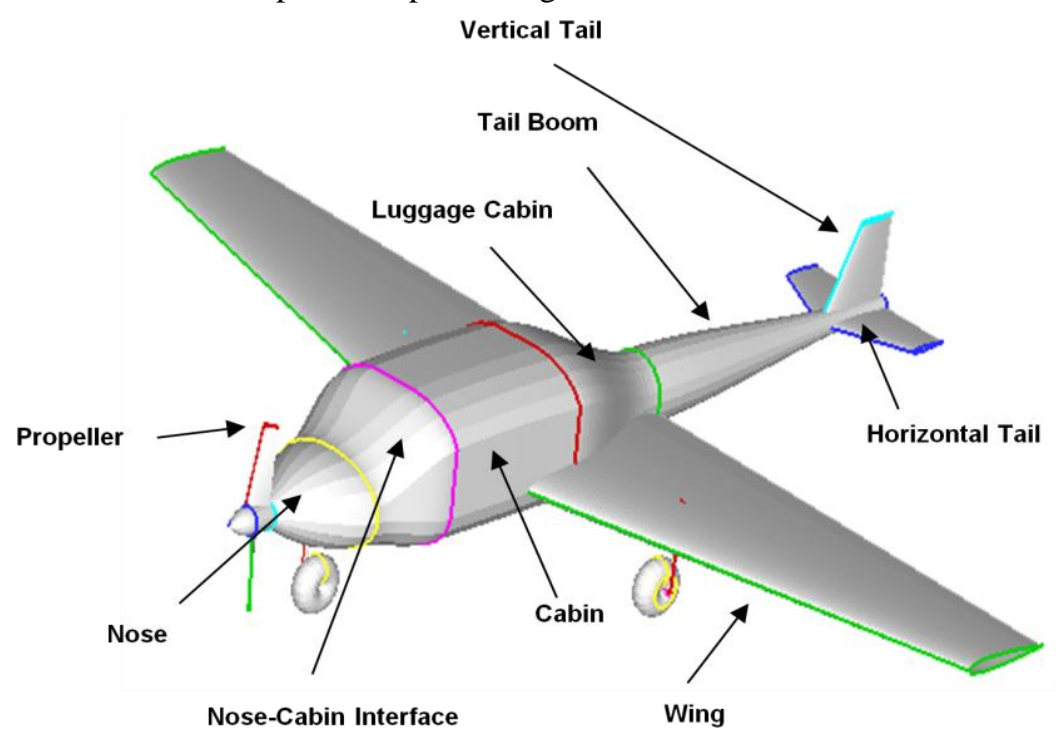

Figure 1 Decomposed aircraft into Components.

\section{SECTIONAL PARAMETRIC DESIGN AND DEVELOPMENT}

To simplify and be generic in the configuration design, the configuration of the aircraft is decomposed or disintegrated into components or objects, which are defined by some design parameters. The components identified are: Fuselage (Nose, Nose-cabin interface, Cabin, Luggage-cabin, Tail-Boom), Empennage (Horizontal tail, Vertical Tail), Wing. The components are defined by design parameters such that a change in a value may change the configuration. Other kinds of parameters used to describe a component are fixed parameters. A fixed parameter is such that for a given desired configuration, its value remains constant while the design parameters may be varied. Examples of the fixed parameters are the nose tip diameter for the nose, dihedral and sweep angles for the wing and the angle of attack. The design parameters are used in creating the geometry models such that with a given parameter, unique and specific class of aircraft could be generated.

The system consists of three major parts namely wing, empennage and fuselage. The system takes the design variables and distributed them to respective parts. Other distinct parts of the system are numerical wind tunnel and surface integrator. The numerical wind tunnel components is used for estimating the surface properties and the surface integrator used for evaluating surface and projected areas of aircraft components. Lift and drag performance is determined for each component and these are then summed to obtained the aircraft lift and drag performance. Components of fuselage are independently created but are joined to create fuselage profile before wind tunnel experiment after which the surface properties of each component are respectively assigned.

\section{DESIGN PARAMETERS}

The design parameters, $\alpha$, are defined as ratios of various geometric lengths to aircraft length. As an example, the nose is completely described by one fixed parameter and three design parameters $\square_{1}, \square_{2}$ and $\square_{3}$, and variation of the design parameters may change the configuration. The nose-cabin interface is defined by three parameters; the cabin by one parameter; the luggage cabin is defined by two parameters and the tail-boom by one parameter. The components of the wing and the empennage are defined by three design parameters each. 
Figures 2 to 5 illustrate examples of the design parameters as applied to the components. It should be noted that each object is defined by both design parameters as indicated on the figures and fixed parameters.

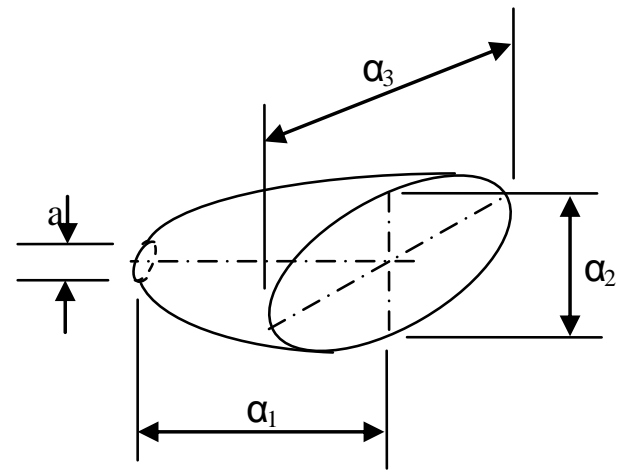

Figure 2: Schematic diagram of Nose

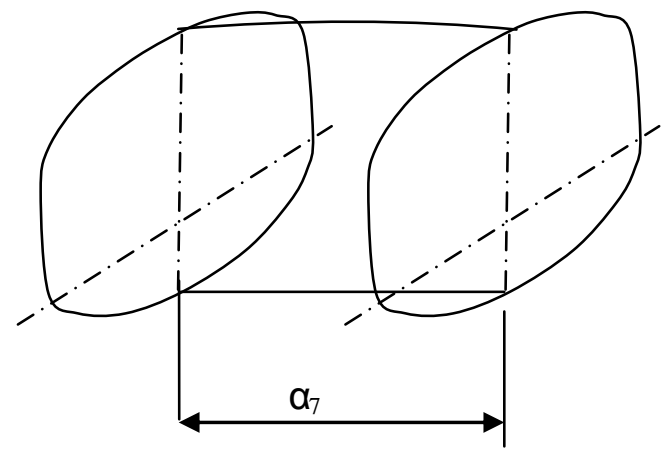

Figure 4: Schematic diagram of Cabin

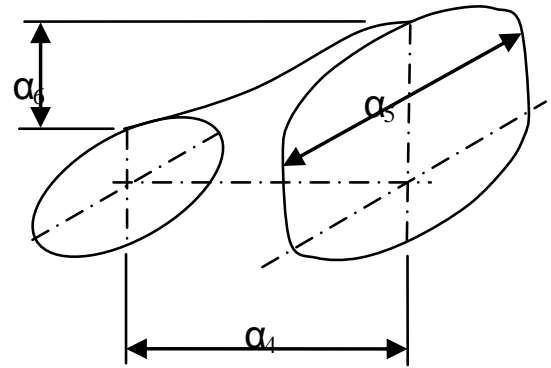

Figure 3: Schematic diagram of Nose-Cabin Intercept

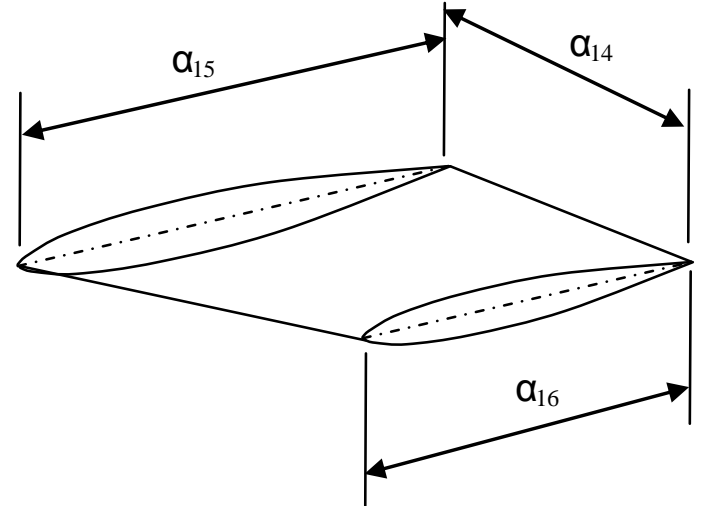

Figure 5: Schematic diagram of Vertical Tail

\section{AERODYNAMIC FORCES ESTIMATION}

The aerodynamic forces arise from two basic sources: 1) Pressure distribution over the body surface and 2) Shear stress distribution over the body surface (Anderson, 2005). The net effect of the pressure and shear stress distributions integrated over the complete aircraft surface gives the resultant aerodynamic force and moment on the aircraft. The resultant force, in Cartesian coordinate system can be resolved into components as lift (aerodynamic force component acting perpendicular to free airstream direction), drag (aerodynamic force component in free airstream direction) and 'side force'. Due to the symmetric nature of aircraft, the side force usually cancels out and does not play significant role in the performance of the aircraft.

\subsection{Pressure Distribution and Pressure Coefficient}

The solution to the Inviscid Flow portion approximation to Navier-Stokes equation results in the velocity of the fluid flow along the object boundary. For incompressible flow, pressure coefficient, $\mathrm{Cp}$, can be expressed in terms of the velocity only. By Bernoulli's equation

$$
P-P_{\infty}=\frac{\rho\left(V_{\infty}^{2}-V^{2}\right)}{2} \quad \text { or } \quad P-P_{\infty}=\frac{1}{2} \rho V_{\infty}^{2}\left(1-\frac{V^{2}}{V_{\infty}^{2}}\right)
$$

where $V$ is the velocity on the object boundary and $V_{\infty}$ is the free stream velocity of flow.

The Pressure coefficient, $\mathrm{Cp}$, is given by $C p=\frac{P-P_{\infty}}{q_{\infty}}$

where $\mathrm{q}_{\infty}$ is the dynamic pressure and $q_{\infty}=\frac{\rho_{\infty} V_{\infty}^{2}}{2}$ 


$$
C p=\frac{P-P_{\infty}}{q_{\infty}}=\frac{\frac{1}{2} \rho\left(V_{\infty}^{2}-V^{2}\right)}{\frac{1}{2} \rho_{\infty} V_{\infty}^{2}} \quad \rightarrow \quad C p=1-\left(\frac{V}{V_{\infty}}\right)^{2}
$$

Therefore $\quad P=\frac{1}{2} \rho V_{\infty}^{2} C p+P_{\infty}$

Average Pressure is evaluated as

$$
P_{\text {avg }}=\frac{\sum_{i=1}^{N} P_{i}}{\text { Number of point } s(N)}
$$

where

$$
\mathrm{i}=1,2, \ldots, \mathrm{N}
$$

\subsection{Shear Stress Distribution}

A viscous, Newtonian fluid (including air and water) moving along a solid boundary will incur a shear stress on that boundary. The no-slip condition dictates that the speed of the fluid at the boundary (relative to the boundary) is zero, but at some distance from the boundary the flow speed must equal that of the free stream. The region between these two points is called the boundary layer. The shear stress is imparted onto the boundary as a result of the loss of velocity and can be expressed as

$$
\tau_{\text {wall }}=\left.\mu \frac{d u}{d y}\right|_{y=0}
$$

where $\mu$ is the dynamic viscosity of the fluid, $u$ is the velocity of the fluid along the boundary, and $y$ is the height of the boundary.

Blasius investigated the shear stress on a plate, and computed for drag forces on flat plate. His result yielded the friction coefficient as function of the Reynolds Number, Re.

$$
C_{f}=f(\mathrm{Re})
$$

The skin friction coefficient is the non-dimensional wall parameter defined as:

$$
C_{f}=\frac{\tau_{\text {wall }}}{\frac{1}{2} \rho V^{2}}
$$

The skin friction coefficient distribution along the streamlines that form the airfoil or fuselage configuration is evaluated using approximation to a flat plate solution of the boundary layer equation known as Blasius solution. For laminar flow, the local skin friction is given by (Anderson, 2005):

$$
c_{f}=\frac{0.664}{\sqrt{\operatorname{Re}_{s}}}
$$

where $\mathrm{Re}_{\mathrm{s}}$ is the local Reynolds number defined as $\quad \operatorname{Re}_{s}=\frac{\rho_{\infty} v_{\infty} s}{\mu_{\infty}}$

where $\mathrm{V}_{\infty}, \mu_{\infty}$ and $\rho_{\infty}$ are freestream quantities and $s$ is the local distance measured from the leading edge. In the case of turbulent flow, the local skin friction is evaluated in accordance with the relationship given as (Houghton and Carpenter, 2003):

$$
c_{f}=\frac{0.0592}{\left(\operatorname{Re}_{s}\right)^{0.2}}
$$

The local shear stress is found to be a function of the skin friction coefficient and is calculated with the equation: $\quad \tau=\frac{C_{f} \rho V^{2}}{2}$

\subsection{Aerodynamic Force Analysis}

The evaluation of the inviscid force on a given aircraft configuration involves the evaluation of the following expressions as they are applied to the geometric surfaces, 


$$
\vec{F}_{P}=\oiint_{\text {Aircraft }} P d \vec{S}
$$

and

$$
\vec{F}_{P}=D_{P} \vec{i}+G_{P} \vec{j}+L_{P} \vec{k}
$$

where $L_{p}, D_{p}$, and $G_{p}$ are the Lift, Drag, and Slip forces due to pressure respectively and $d S$ (elementary surface area) is defined as:

$$
d \vec{S}=d S_{x} \vec{i}+d S_{y} \vec{j}+d S_{z} \vec{k}
$$

with $S_{x}, S_{y}$, and $S_{z}$ being the area projections onto the yz, xz, and xy planes respectively.

Similarly, the force due to shear stress is evaluated with the following expressions,

$$
\begin{aligned}
\vec{F}_{\tau} & =\oiint_{\text {Aircraft }} \tau d \vec{S} \\
\text { and } & \vec{F}_{\tau}=D_{\tau} \vec{i}+L_{\tau} \vec{j}+G_{\tau} \vec{k}
\end{aligned}
$$

where $L_{\tau}, D_{\tau}$, and $G_{T}$ are the Lift, Drag, and Slip forces due to shear respectively.

Using the mean value theorem, equations 14 and 17 become:

$$
\begin{gathered}
\vec{F}_{p}=\oiint_{\text {Aircraft }} p d \vec{S}=p_{\text {avg }} \oiint_{\text {Aircraft }} d \vec{S}=p_{\text {avg }} \oiint_{\text {Aircraft }}\left(d S_{x} \vec{i}+d S_{y} \vec{j}+d S_{z} \vec{k}\right) \\
\vec{F}_{\tau}=\oiint_{\text {Aircraft }} \tau d \vec{S}=\tau_{\text {avg }} \oiint_{\text {Aircraft }} d \vec{S}=\tau_{\text {avg }} \oiint_{\text {Aircraft }}\left(d S_{x} \vec{i}+d S_{y} \vec{j}+d S_{z} \vec{k}\right)
\end{gathered}
$$

The average Pressure $P_{\text {avg }}$ and shear stress $T_{\text {avg }}$ are as evaluated from equation 6 and 13 respectively.

\subsection{Projected and Wetted Areas}

The aircraft shapes are often not shapes whose area can easily be calculated. The surface is divided into small rectangular units with known coordinate points. Each rectangular unit consists of triangular units (Figure 6). Triangulation method, therefore, is used in evaluating the surface area.

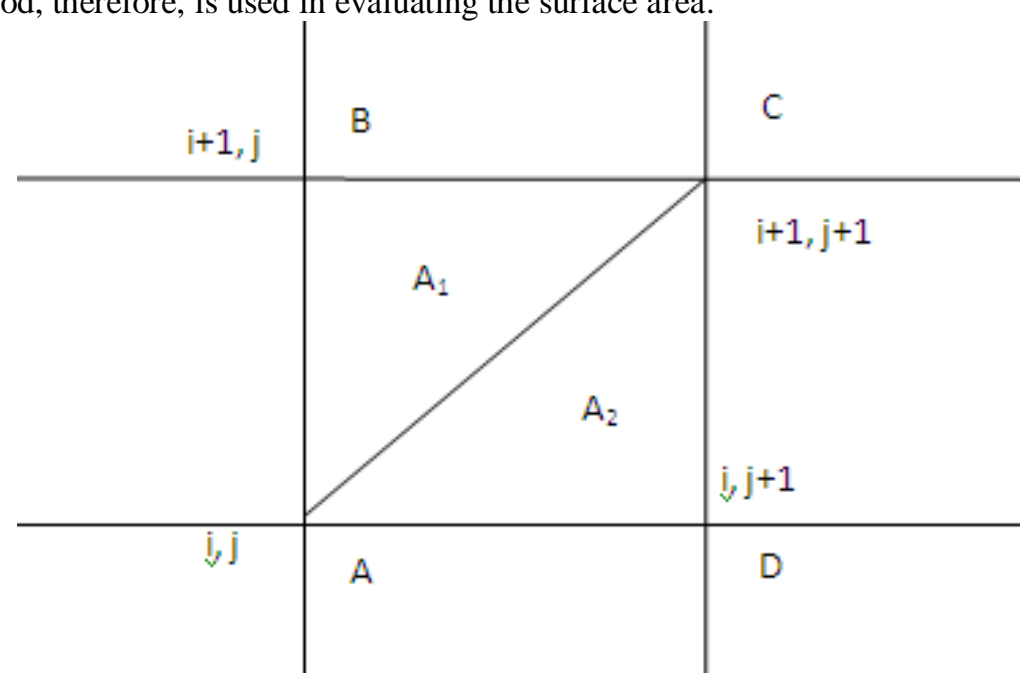

Figure 6: Sample Triangular Element.

With the given coordinates, the lengths of the sides can be calculated, for example, as:

$$
|A B|=\sqrt{\lambda_{m}\left(x_{i+1, j}-x_{i, j}\right)^{2}+\lambda_{m}\left(y_{i+1, j}-y_{i, j}\right)^{2}+\lambda_{m}\left(z_{i+1, j}-z_{i, j}\right)^{2}}
$$

where $\lambda_{m}=0$ if the projection is in the $\mathrm{m}$ direction else $\lambda_{m}=1$.

The area of the triangles is evaluated using the Heron' $s$ formular

$$
\Delta_{\text {area }}=\sqrt{S(S-a b)(S-b c)(S-a c)}
$$

as such $\mathrm{A}_{1}$, area of triangle $\Delta_{1}$ an elementary area dA is

$$
d A=A_{1}=\sqrt{S_{1}\left(S_{1}-A B\right)\left(S_{1}-B C\right)\left(S_{1}-A C\right)}
$$

The area $\mathrm{A}$ is the sum of elementary areas $\mathrm{dA}$ as

$$
A=\sum_{i} d A
$$




\subsection{Aircraft Geometry Results}

\section{RESULTS}

Five set of design parameters are chosen to generate sample plane geometries and the generated aircraft configurations are illustrate in figures 7 to 12 .

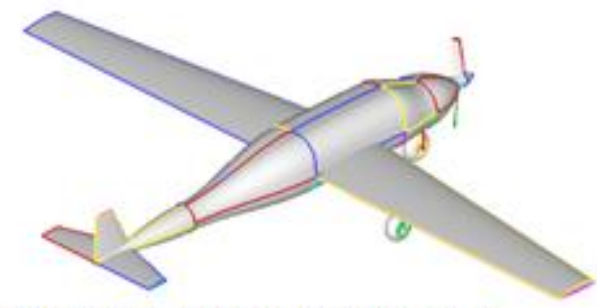

Figure 7: Sample \#1 Configuration.

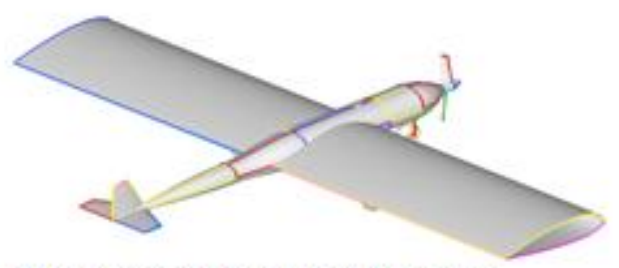

Figure 9: Sample $=3$ Configuration.

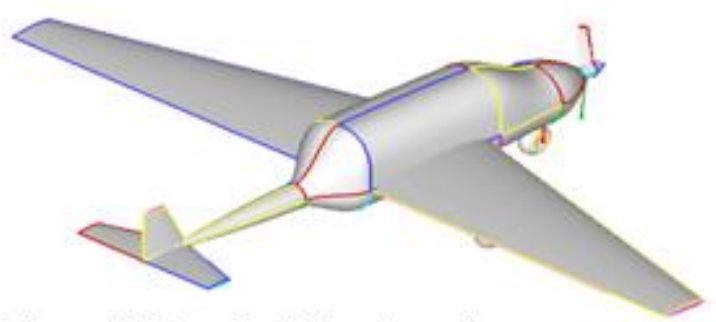

Figure 11: Sample \#5 Configuration.

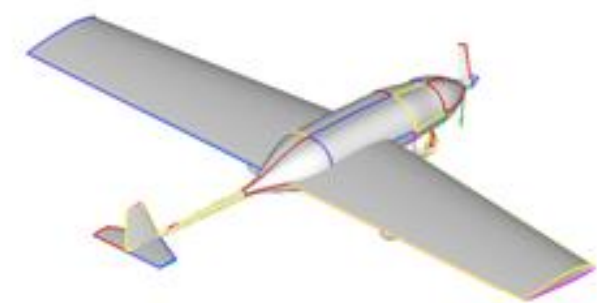

Figure 8: Sample $\# 2$ Configuration.

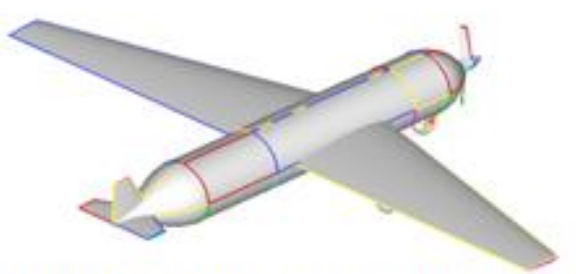

Figure 10: Sample \#4 Configuration.

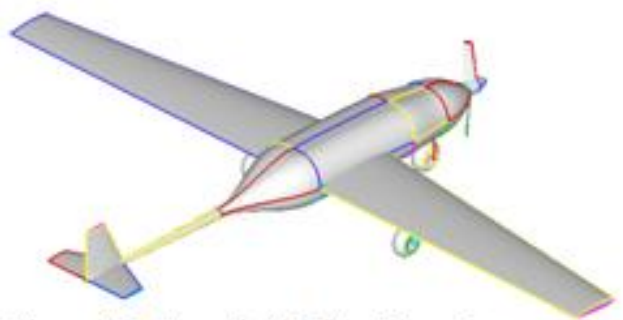

Figure 12: Sample \#7 Configuration.

\subsection{Aerodynamic Performance Results}

The aerodynamics performance of an aircraft is the Lift and Drag developed as the aircraft moves through the air. Five sample aircraft geometries developed were used for analysis. The performances of these samples are studied with the angle of attack variation. The results are illustrated in figures 13 and 14 . From figure 13, the lift performance increases steadily with angle of attack. Sample \#2 shows highest lift performance while sample \#1 indicates least lift performance. The differences may be attributed to differences in wing profiles used. From figure 14, drag performance shows significant variation. Samples \#1 and \#3 indicate an increase in drag performance to maximum then followed by a decrease. Samples \#2, \#4 and \#5, however, show decreasing drag performances to minimum then followed by steady increase. The significant differences in the drag performance may be due to wing or fuselage profile differences.

Configurations of samples \#1 to \#5 were again developed as samples \#6 to \#10, but with same wing profile of sample \#1 (NACA 1412). Lift performance results for samples \#6 to \#10 increase with angle of attack (figures 15). Since the wing profiles are the same, the differences in lift may be attributed to contribution from fuselage components shape differences. Drag performance results indicate similar trend as shown in figure 16 (compared with samples \#1 to \#5) but with sample \#8 indicating higher drag performances. This infers that the nature of the drag performance is not influenced significantly by the wing (figure 16). The magnitude of the drag however, may be affected by different wing profiles. This is illustrated by the case of sample \#6 (similar to \#1) becoming higher than that of sample \#8 (similar to \#3 in figure 16) which is in contrast with when different wing profiles were used (figure 14). 


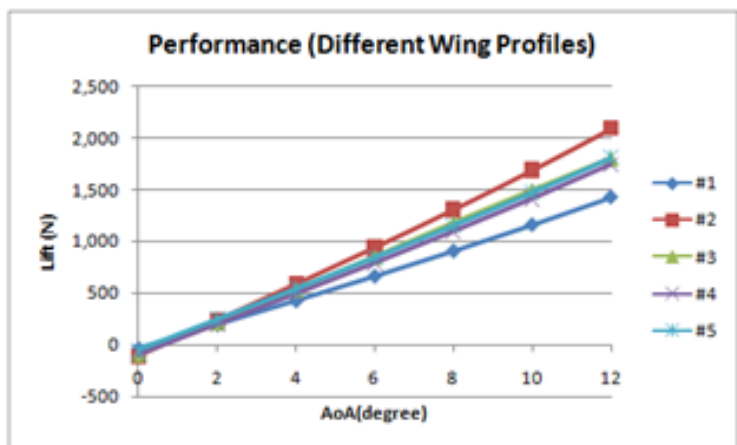

Figure 13: Lift Performance with different wing profile.

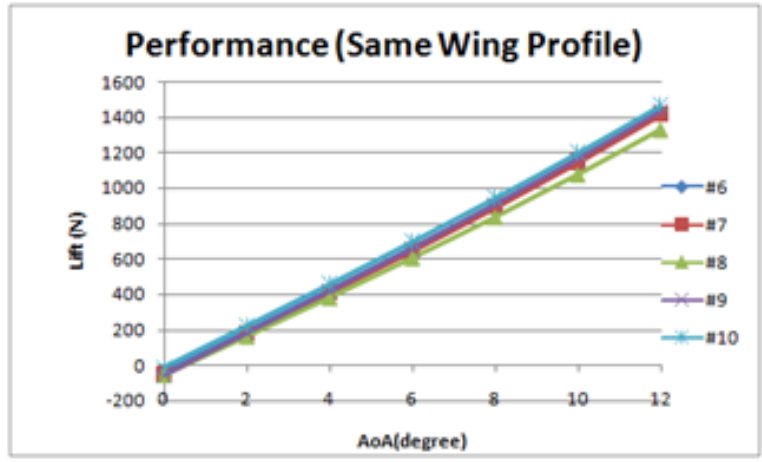

Figure 15: Lift Performance with the same wing profile.

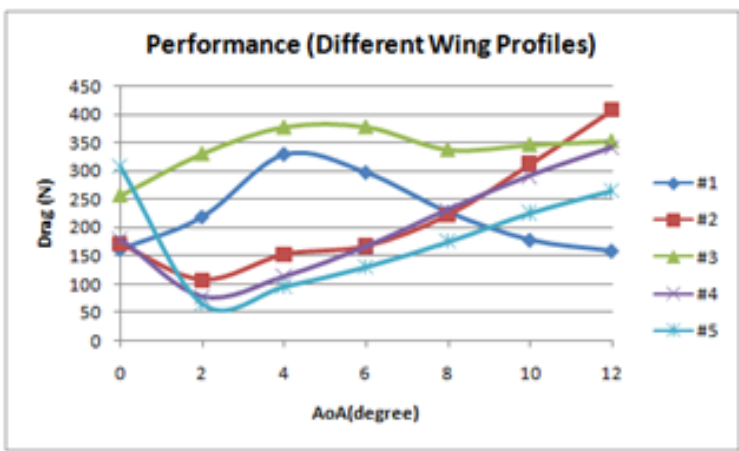

Figure 14: Drag Performance with different wing profiles.

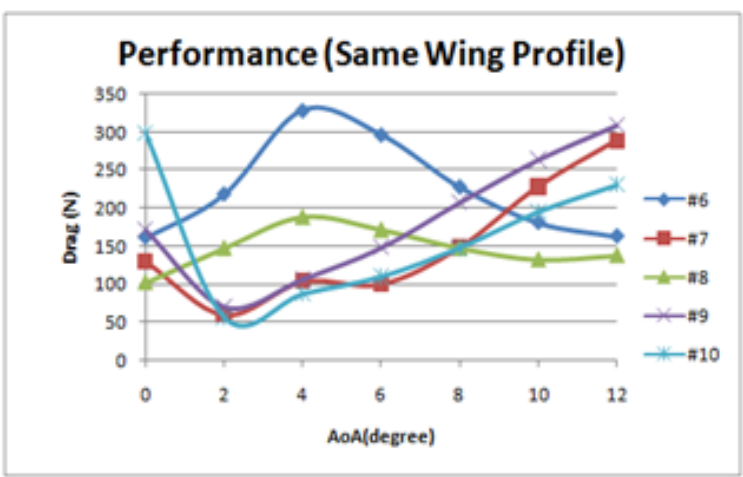

Figure 16: Drag Performance with the same wing profile.

The components or objects of the aircraft are expected to contribute to lift and drag performance of the aircraft. Figure 17 is the plot of lift performance of each component contributed toward the overall lift performance of the aircraft of sample \#1. The figure shows that major contributor to lift is the wing as expected. It is noted that the contribution by luggage cabin object decreases with increase in angle of attack. Sample \#2 also indicates similar trend for the lift performance.

The drag performance contributions from various object components are of varying nature. From figure 18 , drag performance of the wing increases with increase in angle of attack as expected. Notable component is the luggage cabin drag performance. For sample \#1 the drag force of luggage cabin increases to a maximum then decreases. However, for sample \#2, luggage cabin drag force increases with angle of attack (figure 20).

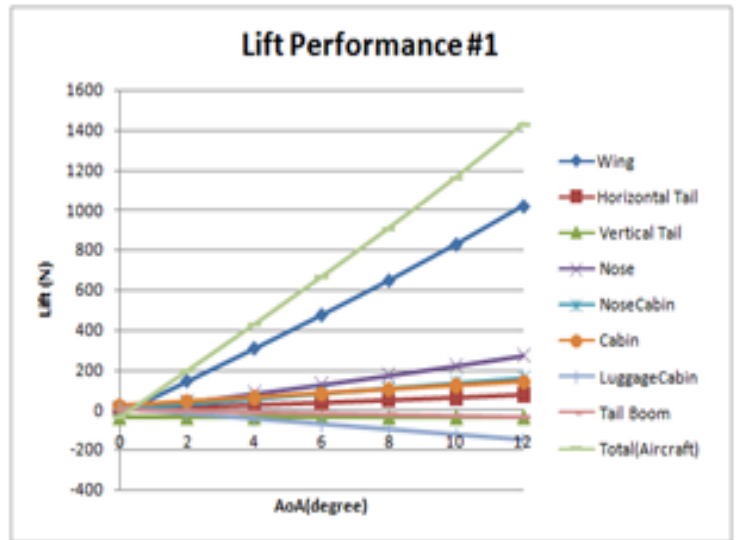

Figure 17: Object Lift Performance for Sample $\# 1$.

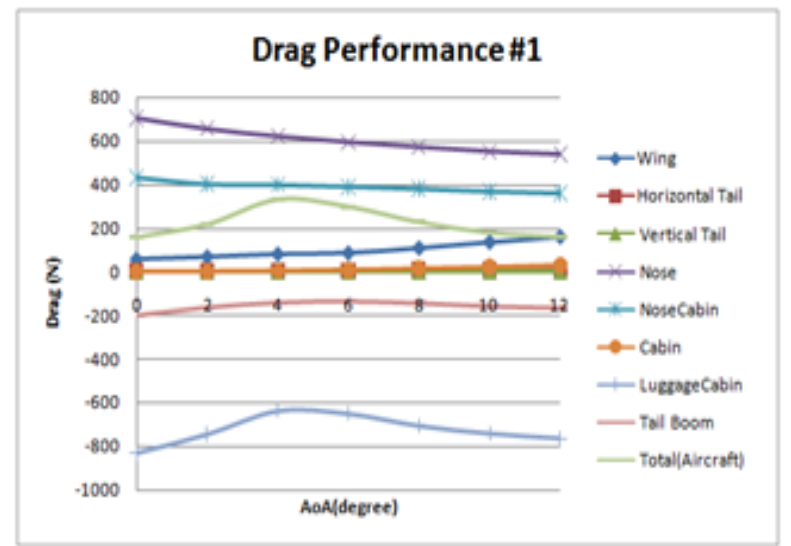

Figure 18: Object Drag Performance for Sample $\# 1$. 


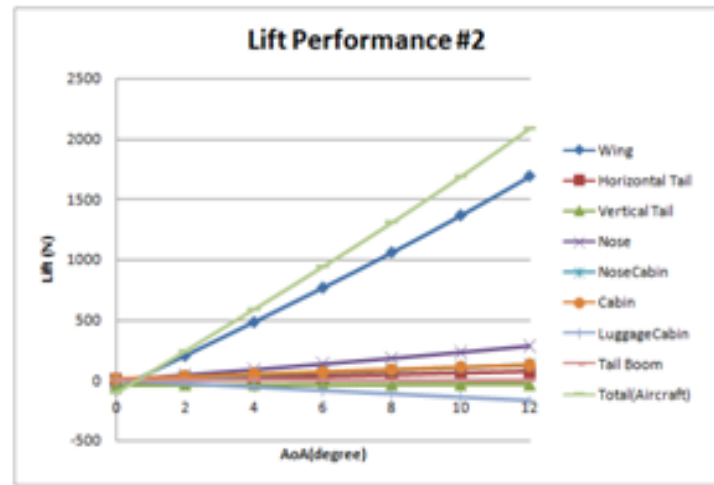

Figure 19: Object Lift Performance for Sample $\# 2$.

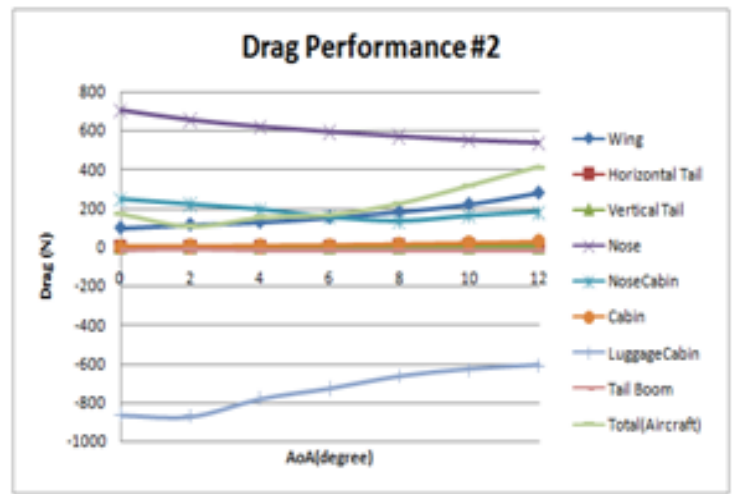

Figure 20: Object Drag Performance for Sample $=2$.

\section{CONCLUSION}

There were 19 design variables identified that completely define aircraft configuration. Ten sample aircraft geometries have been developed with some chosen design parameters. Five of these samples were of the same wing profile. The aerodynamic performance results of the samples, generally shows increase in lift performance with increase in angle of attack. The drag performance indicates varying results with increase in angle of attack. The lift performance of the samples with same wing profile show that the components of the fuselage contributed towards the lift force. These contributions indicated higher (from 10\% at angle of attack of $2^{\circ}$ ) overall lift force compared to lift force developed by the wing alone. Hence, the estimated lift performance is higher when all surfaces are considered. The total lift and drag estimate of an aircraft using the developed system is more accurate than using estimate from the wing alone.

With this tool, aerodynamic performance of the aircraft, usually estimated from the lift and drag coefficients obtained as a result of the selected airfoil of the wing geometry, will be better estimated more accurately with contribution from all surfaces.

\section{REFERENCES}

[1] Anderson Jr, John D., (2005), "Fundamentals of Aerodynamics", McGraw-Hill Companies, Inc., New York

[2] Anderson Jr, John D., (1995), "Computational Fluid Dynamics: The Basics with Applications”, McGrawHill Companies, Inc., New York

[3] Antoine, Nicolas E. and Kroo, Ilan M. (2005), "Framework for Aircraft Conceptual Design and Environmental Performance Studies”, AIAA JOURNAL, Vol. 43, No. 10, October 2005

[4] David L. Rodriguez and Peter Sturdza, (2006), "A rapid Geometry Engine for Preliminary Aircraft Design", 44th AIAA Aerospace Sciences Meeting and Exhibit, 9 - 12 January 2006, Reno, Nevada.

[5] Gloudemans, J. R., Davis, Paul C., and Gelhausen, Paul A., (1996), "A rapid geometry modeler for Conceptual Aircraft", AIAA-1996-0052, January, 1996

[6] Grigg, Alan; Audsley, Neil; Fletcher, Martyn and Wake, Allan, "A Method for Design and Analysis of Next Generation Aircraft Computer Systems"

[7] Houghton, E.L. and Carpenter, P.W. (2003), “Aerodynamics for Engineering Students", $5^{\text {th }}$ Edition, Butterworth - Heinemann,

[8] Kroo,Ilan; Altus, Steve; Braun, Robert; Gage, Peter and Sobieski, Ian "Multidisciplinary Optimization Methods for Aircraft Preliminary Design", AIAA 94-4325

[9] Labrujere, Th.E. and Slooff, J.W. (1993), "Computational Methods for the Aerodynamic Design of Aircraft Components", Annu. Rev. Fluid Mech. 25: 183-214

[10] Neufeld, Daniel and Chung, Joon (2007), "Development of Aircraft Conceptual Design Optimization Software", Fifth International Conference on Computational Science and Applications, IEEE

[11] Raymer, Daniel P., (2002), "Enhancing Aircraft Conceptual Design using Multidisciplinary Optimization", Doctoral Thesis, Swedish Royal Institute of Technology, 2002

[12] Stinton, Darrol (1998), "The Anatomy of the Aeroplane", Blackwell Science Ltd., Oxford

[13] Turevskiy, A. Gage, S. and Buhr, C (1999), "Model-Based Design of a new light-weight Aircraft", American Institute of Aeronautics and Astronautics. 